\title{
A novel IncRNA, TCONS_00006195, represses hepatocellular carcinoma progression by inhibiting enzymatic activity of ENO1
}

Songman $\mathrm{Yu}^{1}$, Ning $\mathrm{Li}^{2}$, Zebing Huang ${ }^{1}$, Ruochan Chen ${ }^{1}$, Panpan $\mathrm{Yi}^{1}$, Rui Kang ${ }^{3}$, Daolin Tang $^{3}$, Xingwang Hu${ }^{1}$ and Xuegong Fan ${ }^{1}$

\begin{abstract}
Hepatocellular carcinoma (HCC) is one of the most common malignancies and has an unfavorable prognosis. The hepatitis B virus $X(\mathrm{HBx})$ protein has been reported to be closely associated with hepatocarcinogenesis. Meanwhile, emerging evidence has indicated that long noncoding RNAs (IncRNAs) are involved in the pathogenesis and progression of cancers. Our previous investigation has demonstrated that HBx could promote HCC by regulating the expression levels of various IncRNAs. In this study, we identified an IncRNA, IncRNA-TCONS_00006195 (termed IncRNA6195), which was downregulated in HBV-related HCC tissues compared with its expression in adjacent noncancerous hepatic tissues. Clinical data showed that a low level of IncRNA-6195 was correlated with a high Edmondson-Steiner grade of the tumor and a poor prognosis in HCC patients. Furthermore, IncRNA-6195 acted as a tumor repressor in the development of hepatitis B-related HCC, inhibiting HCC cell proliferation in vitro and in vivo. Moreover, IncRNA-6195 could combine with a-enolase (ENO1) and repress its enzymatic activity, thus further inhibiting the energy metabolism in HCC cells. Our results suggest that IncRNA-6195 represses the growth of HCC by inhibiting the enzymatic activity of ENO1. These findings provide new insights into the mechanisms underlying the IncRNA involvement in hepatocarcinogenesis and can serve as a basis for the development of novel strategies to hinder HCC.
\end{abstract}

\section{Introduction}

Hepatocellular carcinoma (HCC) is one of the most common human malignancies and the third leading cause of cancer-related deaths worldwide ${ }^{1}$. Chronic hepatitis B virus (HBV) infection is the major cause of HCC in China. Although researchers have determined some factors contributing to HBV-induced HCC tumorigenesis, such as genomic instability, insertional mutagenesis, and

\footnotetext{
Correspondence: Xingwang Hu (lebithu@csu.edu.cn) or

Xuegong Fan (xgfan@hotmail.com)

'Department of Infectious Diseases, Hunan Key Laboratory of Viral Hepatitis,

Xiangya Hospital, Central South University, Changsha, China

2Department of Blood Transfusion, Xiangya Hospital, Central South University,

Changsha, China

Full list of author information is available at the end of the article.

Edited by A Stephanou.
}

epigenetic changes ${ }^{2,3}$, the underlying molecular mechanisms are still unclear.

Long noncoding RNAs (lncRNAs) are a class of transcripts that have more than 200 nucleotides and exhibit no protein-coding potential. Recently, emerging evidence has indicated that IncRNAs play critical roles in the pathogenesis and progression of cancers ${ }^{4}$. A number of lncRNAs, such as ATB (lncRNA activated by transforming growth factor- $\beta)^{5}$, DANCR (differentiation-antagonizing non-protein-coding RNA) ${ }^{6}$, HEIH (lncRNA highly expressed in $\mathrm{HCC})^{7}$, MVIH (lncRNA associated with microvascular invasion in $\mathrm{HCC})^{8}$, and TP73-AS1 (P73 antisense RNA 1T) ${ }^{9}$, have been found to be dysregulated in and associated with HCC. These lncRNAs participate in various biological processes, including cell

\section{(c) The Author(s) 2018}

(c) (i) Open Access This article is licensed under a Creative Commons Attribution 4.0 International License, which permits use, sharing, adaptation, distribution and reproduction cc. in any medium or format, as long as you give appropriate credit to the original author(s) and the source, provide a link to the Creative Commons license, and indicate if changes were made. The images or other third party material in this article are included in the article's Creative Commons license, unless indicated otherwise in a credit line to the material. If material is not included in the article's Creative Commons license and your intended use is not permitted by statutory regulation or exceeds the permitted use, you will need to obtain permission directly from the copyright holder. To view a copy of this license, visit http://creativecommons.org/licenses/by/4.0/. 


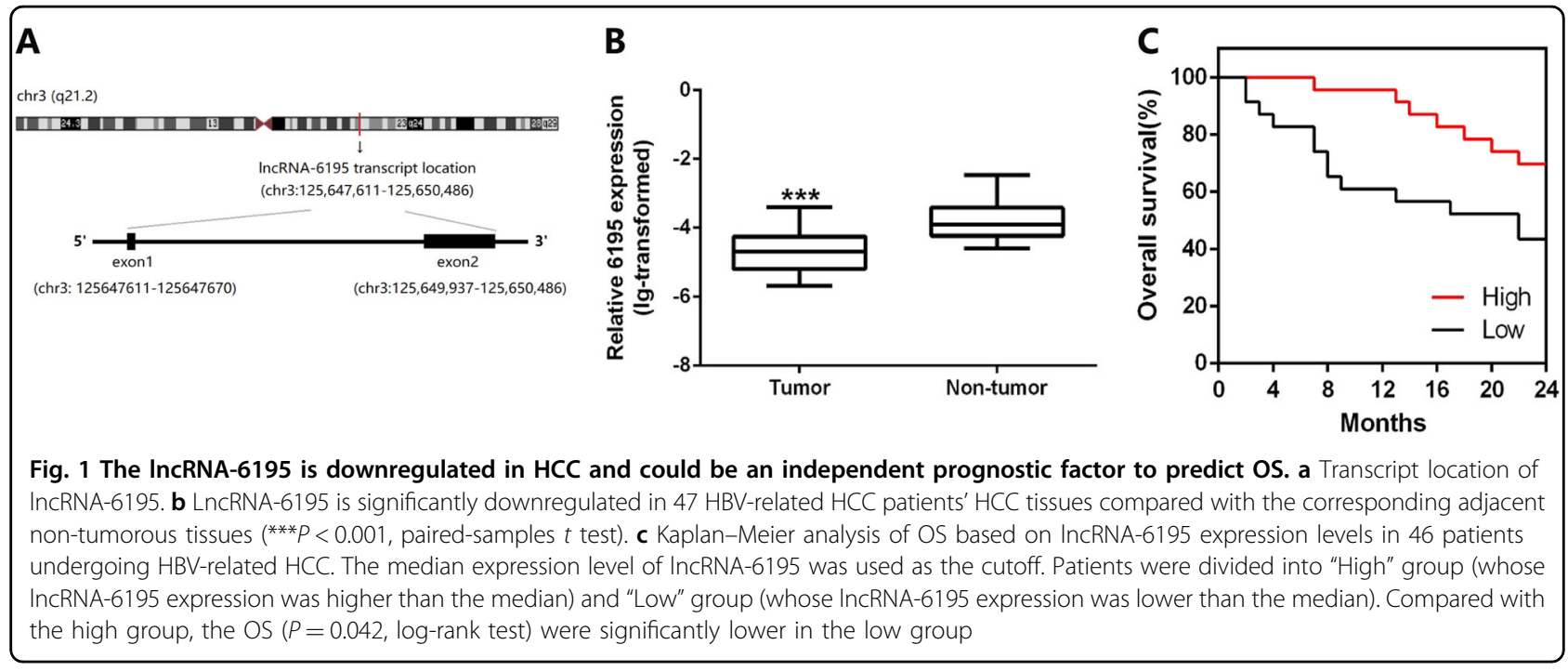

proliferation, apoptosis, invasion, and migration ${ }^{10}$. The HBV X $(\mathrm{HBx})$ protein has been reported to be closely associated with $\mathrm{HBV}$-induced hepatocarcinogenesis. In recent years, some lncRNAs, such as DREH ${ }^{11}, \mathrm{UCA}^{12}$, and Unigene $56159^{13}$, have been proven to be regulated by $\mathrm{HBx}$ and involved in the pathogenesis and progression of HBV-related HCC. However, the functions and mechanisms of most HBx-related lncRNAs in HCC are still unclear.

$\alpha$-Enolase (ENO1) is an enolase isoform present in almost all adult tissues in mammals. It was originally characterized as a key enzyme of glycolysis, catalyzing the conversion of 2-phosphoglycerate (2PG) to phosphoenolpyruvate $(\mathrm{PEP})^{14}$. After decades of research, scientists have demonstrated that besides its glycolytic function in normal processes, ENO1 also participates in several critical biological processes in cancer, including proliferation, migration, and invasion ${ }^{15-18}$.

In our previous study, we have used an IncRNA hybridization-based microarray and real-time polymerase chain reaction (PCR) to obtain the lncRNA expression profiles of L02/HBx and L02/pcDNA3.0 cell lines. In this study, we further investigated the biological function and the underlying mechanism of an $\mathrm{HBx}$-upregulated lncRNA, lncRNA-TCONS_00006195 (termed lncRNA6195), in vivo and in vitro to potentially find a new strategy to treat HCC.

\section{Results}

\section{LncRNA-6195 is downregulated in HCC tissue}

In our previous study ${ }^{19,20}$, we have found that compared with the control group, which was stably transfected with a blank plasmid (L02/pcDNA3.0), LO2/HBx cells had 323 upregulated and 421 downregulated lncRNAs (fold change $>2.0, P<0.05)$. Expression of IncRNA-6195 was one of the most significantly altered in $\mathrm{LO} 2 / \mathrm{HBx}$ cells, and we speculated that lncRNA-6195 might play an important role in HCC. The lncRNA-6195-coding sequence, consisting of exons 1 and 2 , is located on chromosome 3 , between oxysterol-binding protein-related protein $11(55,137 \mathrm{bp})$ at the $5^{\prime}$-end and translation initiation factor IF-2-like isoform X1 (105,706 bp) at the 3 '-end (Fig. 1a). By searching for the lncRNA-6195 sequence in LNCipedia (https://lncipedia.org), we found that the PhyloCSF ${ }^{21}$ score of lncRNA-6195 was -83.9101 , and the CPAT ${ }^{22}$ coding probability was $1.78 \%$. The analysis result of PRIDE reprocessing $2.0^{23}$ was zero. Additionally, LNcipedia failed to predict any Bazzini small open reading frames ${ }^{24}$ or Lee translation initiation sites ${ }^{25}$ in lncRNA-6195. All these data suggest that the lncRNA6195 transcript is consistent with an lncRNA.

To investigate the expression levels of lncRNA-6195 in HBV-related HCC, quantitative reverse transcription-PCR (qRT-PCR) was performed on 47 pairs of human HBVrelated HCC tissues and matched non-tumorous liver tissues. The transcript levels of lncRNA-6195 were significantly downregulated in HCC tissues compared with those in adjacent non-tumorous hepatic tissues $(P<0.001$; Fig. 1b). Additionally, the expression level of HBx in HCC tissue also significantly reduced compared with adjacent non-tumor tissues and the expression of lncRNA-6195 and $\mathrm{HBx}$ was positively correlated in HCC tissue (data not shown). Further, clinical data of these 47 patients showed that the low level of lncRNA-6195 was correlated with a high Edmondson-Steiner grade of the tumor $(P<0.05$; Table 1). Kaplan-Meier analysis of 46 HBV-related HCC patients (one patient who died from postoperative massive hemorrhage was excluded) revealed that lower lncRNA-6195 expression levels in HCC tissues significantly correlated with a markedly reduced overall 
Table 1 Relationship between IncRNA-6195 expression and the clinical characteristics of HCC patients

\begin{tabular}{|c|c|c|c|}
\hline & \multicolumn{2}{|c|}{ LncRNA-6195 expression levels } & \multirow[t]{2}{*}{$P$ value } \\
\hline & $\begin{array}{l}\text { Low } \\
\text { expression }\end{array}$ & $\begin{array}{l}\text { High } \\
\text { expression }\end{array}$ & \\
\hline All cases & 23 & 24 & \\
\hline \multicolumn{4}{|l|}{ Age (years) } \\
\hline$\leq 55$ & 18 & 17 & 0.559 \\
\hline$>55$ & 5 & 7 & \\
\hline \multicolumn{4}{|l|}{ Gender } \\
\hline Male & 22 & 23 & 0.975 \\
\hline Female & 1 & 1 & \\
\hline \multicolumn{4}{|c|}{ HBV-DNA (copies/ } \\
\hline$\leq 10^{3}$ & 18 & 13 & 0.081 \\
\hline$>10^{3}$ & 5 & 11 & \\
\hline \multicolumn{4}{|c|}{ Tumor size $(\mathrm{cm})$} \\
\hline$\leq 5$ & 11 & 10 & 0.671 \\
\hline$>5$ & 12 & 14 & \\
\hline \multicolumn{4}{|l|}{ Tumors $(n)$} \\
\hline Multiple & 4 & 6 & 0.524 \\
\hline Solitary & 19 & 18 & \\
\hline \multicolumn{4}{|l|}{ Cirrhosis } \\
\hline Yes & 15 & 19 & 0.285 \\
\hline No & 8 & 5 & \\
\hline \multicolumn{4}{|l|}{ AFP $(\mathrm{ng} / \mathrm{ml})$} \\
\hline$\leq 20$ & 6 & 7 & 0.813 \\
\hline$>20$ & 17 & 17 & \\
\hline \multicolumn{4}{|c|}{ Edmondson grade } \\
\hline$|-| \mid$ & 16 & 23 & $0.017^{*}$ \\
\hline III-IV & 7 & 1 & \\
\hline \multicolumn{4}{|c|}{ Vascular invasion } \\
\hline Yes & 7 & 11 & 0.278 \\
\hline No & 16 & 13 & \\
\hline \multicolumn{4}{|c|}{ Capsular invasion } \\
\hline Yes & 12 & 8 & 0.192 \\
\hline No & 11 & 16 & \\
\hline
\end{tabular}

The median expression level of IncRNA-6195 was used as the cutoff. "Low expression" group include 23 patients whose IncRNA-6195 expression was lower than the median. "High expression" group include 24 patients whose IncRNA6195 expression was higher than the median. Pearson's $X$ test and Fisher's exact test were used to analyze the correlation between IncRNA-6195 expression levels and clinical features. ${ }^{*} P<0.05$. survival $(\mathrm{OS})(P=0.042$, log-rank test; Fig. 1c) of the HBV-related HCC patients. These results together suggest that lncRNA-6195 may play an important role in the pathogenesis and prognosis of HBV-related HCC.

\section{LncRNA-6195 inhibits cell proliferation in vitro}

To determine its function, the effects of IncRNA-6195 on cell proliferation, apoptosis, migration, and invasion were investigated by gain- and loss-of-function studies in the L02, HepG2, and Huh7 cell lines. The results showed that overexpression of cellular lncRNA-6195 not only suppressed the cell proliferation (Fig. 2a-d) but also inhibited the migration and invasion activities of liver cancer cells, compared with those in the negative control (Supplementary Fig. S1B-E). However, IncRNA-6195 upregulation had no significant effect on cell apoptosis in vitro (Supplementary Fig. S1F).

The L02 and HepG2 cell lines stably overexpressing lncRNA-6195 were constructed using the pc-6195 plasmid and were verified by RT-PCR (Supplementary Fig. S1A), the CCK-8 assay (Fig. 2a, b), and the colony formation assay (Fig. 2c, d). The results showed that overexpression of lncRNA-6195 could suppress the cell proliferation. Subsequently, cell cycle distribution was investigated by flow cytometry, and the data demonstrated that upregulation of lncRNA-6195 increased the proportion of cells in the G1 phase and inhibited the cell cycle progression (Fig. 2g-i). In addition, the cyclin D1 protein was downregulated in the lncRNA-6195overexpressing L02 and HepG2 cell lines (Fig. 2e, f).

To further confirm these observations, rescue experiments were performed. Knockdown of the expression of lncRNA-6195 (Supplementary Fig. S2A) significantly promoted the cell proliferation of HepG2/6195 (HepG2 cells stably transfected with pc-6195) and Huh7/6195 (Huh7 cells stably transfected with pc-6195) cells (Fig. 3a-d). In addition, inhibition of the lncRNA-6195 expression upregulated the cyclin D1 protein (Fig. 3e, f) and decreased the proportion of HepG2/6195 cells in the G1 phase (Fig. 3g, h). All these results suggest that lncRNA-6195 may inhibit the cell proliferation in vitro.

\section{LncRNA-6195 inhibits tumorigenesis in vivo}

The results obtained in BALB/C nude mice, which were subcutaneously injected with HepG2/6195 or HepG2/pc cells, showed that compared with those in the control group, subcutaneous tumors were significantly smaller in the mice injected with HepG2/6195 cells (Fig. 4a-d). Ki67 immunohistochemistry (IHC) staining revealed lower Ki-67 expression levels in the tumors from the HepG2/ 6195 group compared with those in the tumors from the control group (Fig. 4e, middle and lower panels, and Fig. 4f), which indicated a slower growth of the HepG2/ 6195-derived tumors. However, hematoxylin and eosin 

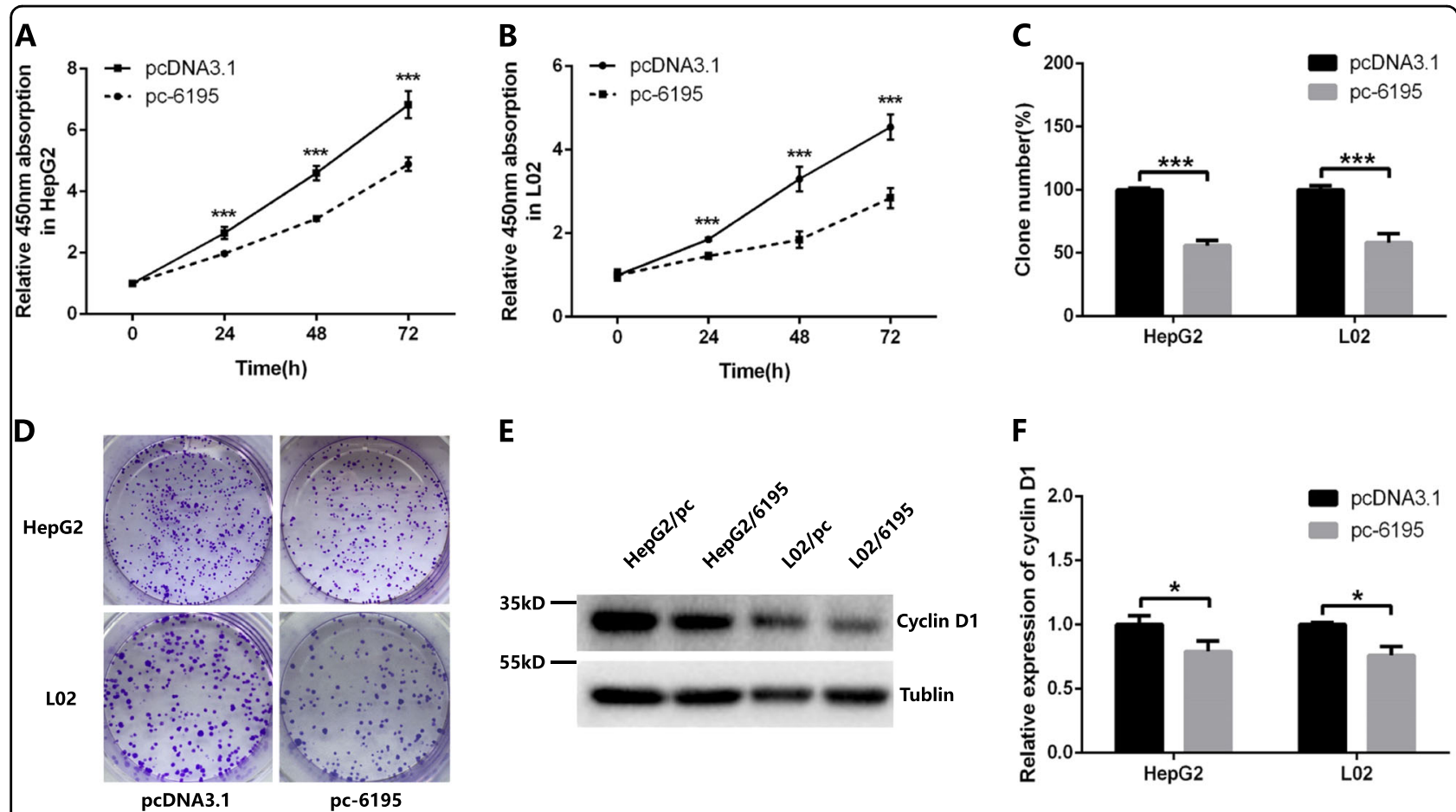

E


H
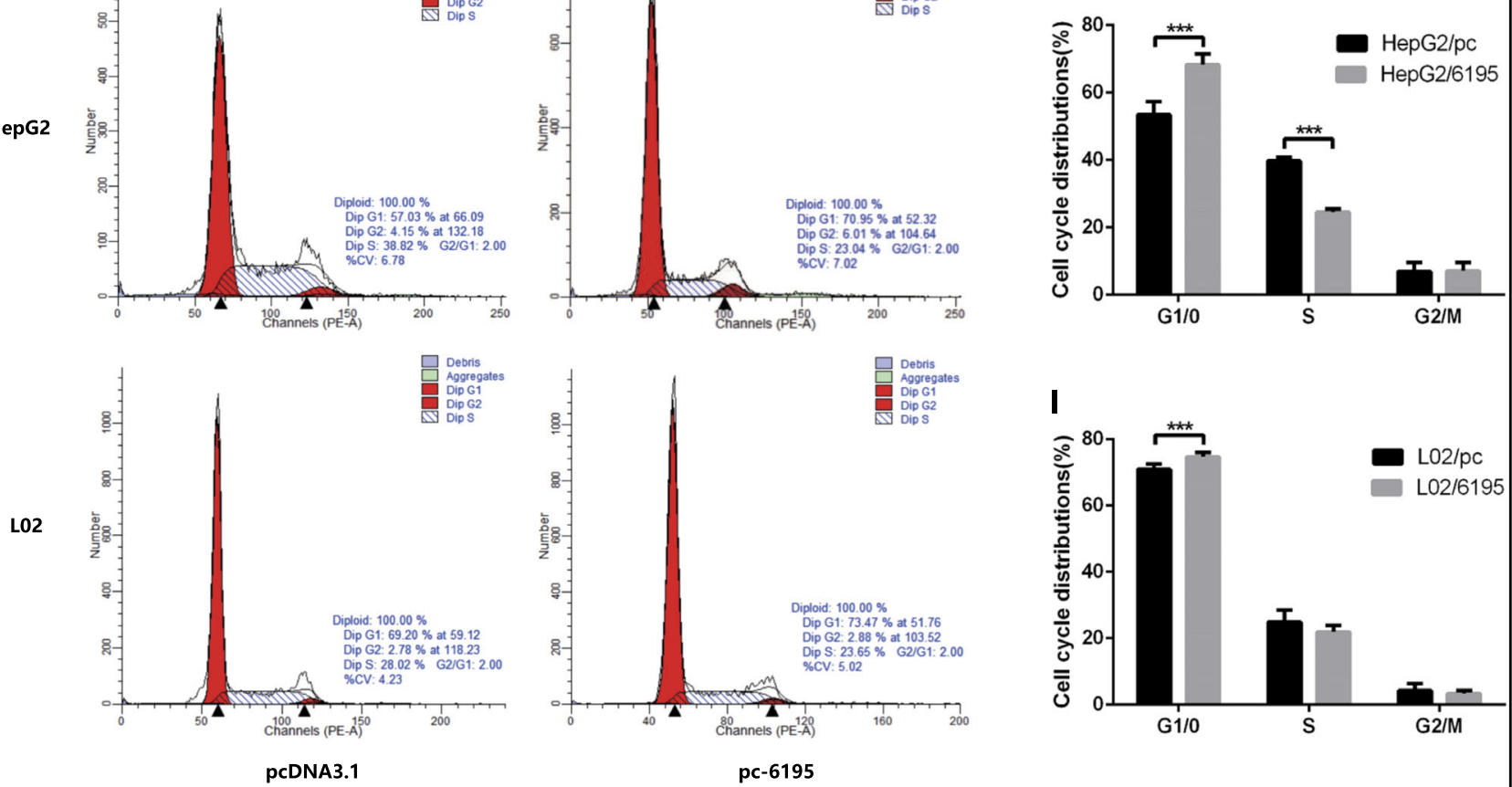

Fig. 2 Overexpression of IncRNA-6195 inhibits cell proliferation in vitro. $\mathbf{a}$, b Overexpression of IncRNA-6195 inhibits cell proliferation in HepG2 and L02 cell lines as assessed by the CCK-8 assay. c, d Overexpression of IncRNA-6195 inhibits cell proliferation in HepG2 and L02 cell lines as assessed by the colony formation assay. e, $\mathbf{f}$ Cyclin D1 protein is downregulated in IncRNA-6195 overexpressed HepG2 and L02 cell lines. $\mathbf{g}$-i Overexpression of IncRNA-6195 increases the cell numbers in the G1 phase and inhibits cell cycle progression in HepG2 and L02 cells. ${ }^{*} P<0.05$, ${ }^{* * *} P<0.001$, Student's $t$ test. Data are represented as mean \pm SD.) 


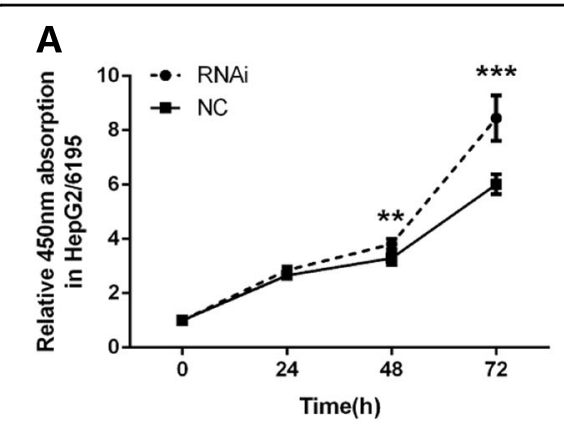

D
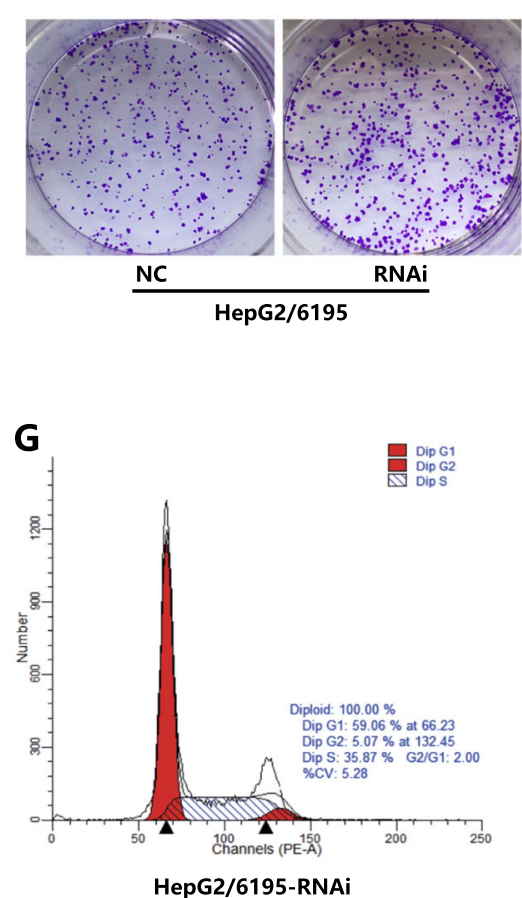

B



$\mathbf{E}$

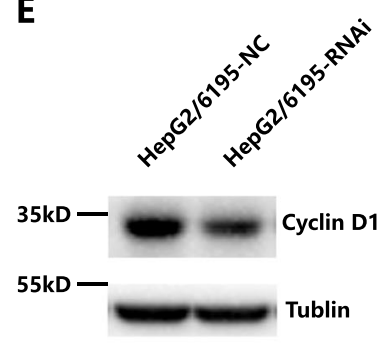

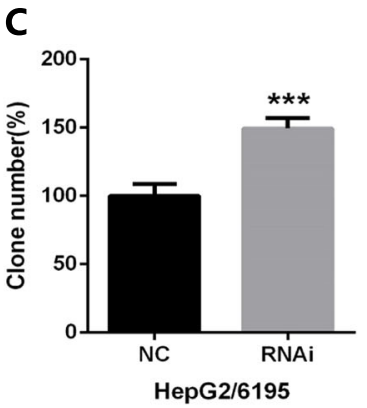

$\mathbf{F}$

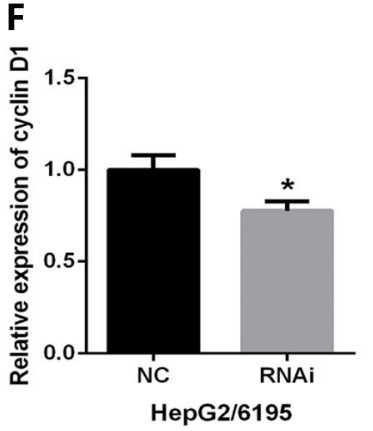

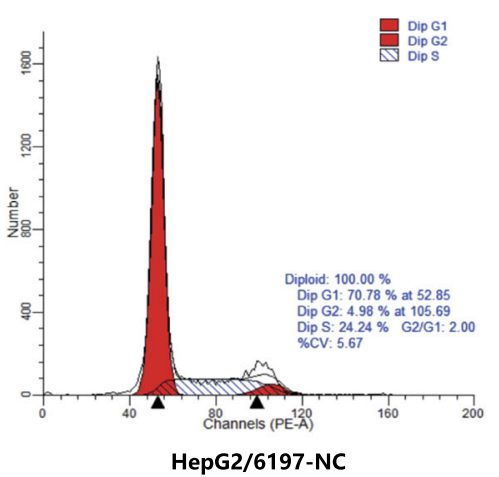

$\mathbf{H}$

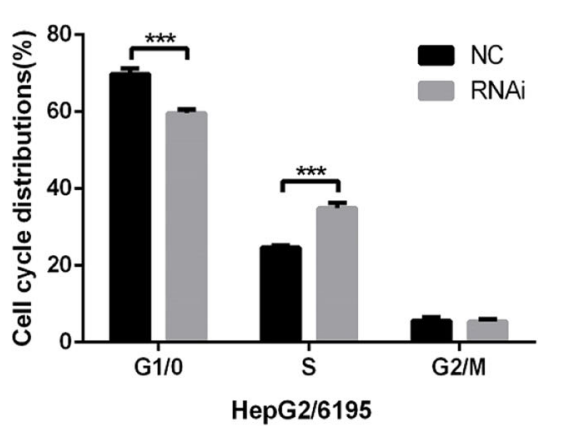

Fig. 3 Inhibition of IncRNA-6195 promotes cell proliferation in vitro. a, b IncRNA-6195 shRNA promoted cell proliferation as assessed by the CCK-8 assay after stable transfection of IncRNA-6195 shRNA(RNAi) or negative control shRNA (NC) in HepG2/6195 (a) and Huh7 cells (b). c, d Inhibition of IncRNA-6195 promotes cell proliferation in HepG2/6195 cells as assessed by the colony formation assay. e, $\mathbf{f}$ Western blot results showed that Cyclin D1 protein is upregulated in IncRNA-6195 knock-downed HepG2/6195 cell lines. $\mathbf{g}$, $\mathbf{h}$ Inhibition of IncRNA-6195 decreases the cell numbers in the G1 phase and promotes cell cycle progression as analyzed using flow cytometry. $\left({ }^{*} P<0.05,{ }^{* *} P<0.01,{ }^{* * *} P<0.001\right.$, Student's $t$ test. Data are represented as mean $\pm \mathrm{SD}$.)

(HE) staining of tumors from the two groups did not show any significant difference (Fig. 4e, upper panel).

\section{LncRNA-6195 can combine with ENO1 protein}

To investigate whether lncRNA-6195 functions by interacting with a specific target, we performed an RNA pull-down assay to identify proteins associated with IncRNA-6195 (Fig. 5a). The precipitated proteins were separated by $12 \%$ sodium dodecyl sulfate-polyacrylamide gel electrophoresis (SDS-PAGE) and then silver-stained. The differentially expressed bands, compared with those in the negative control, were excised to be identified by mass spectrometry (Table 2). The results of mass spectrometry indicated that ENO1 might be specifically associated with lncRNA-6195, and subsequent western blot analysis confirmed these results (Fig. 5b). To further verify the association between lncRNA-6195 and ENO1, we performed a RNA immunoprecipitation (RIP) assay with an antibody against ENO1 and a nonspecific antibody (immunoglobulin G (IgG)) using HepG2 cellular extracts. Consistently, we observed a significantly higher enrichment level of IncRNA-6195 with the ENO1 




antibody than that with IgG (Fig. 5c, d). Thus, the results of both assays demonstrated that lncRNA-6195 could combine with ENO1 in vitro.
To identify the binding sites of IncRNA-6195, we constructed a series of deletion mutants of lncRNA-6195 and performed an RNA pull-down assay to map the ENO1- 


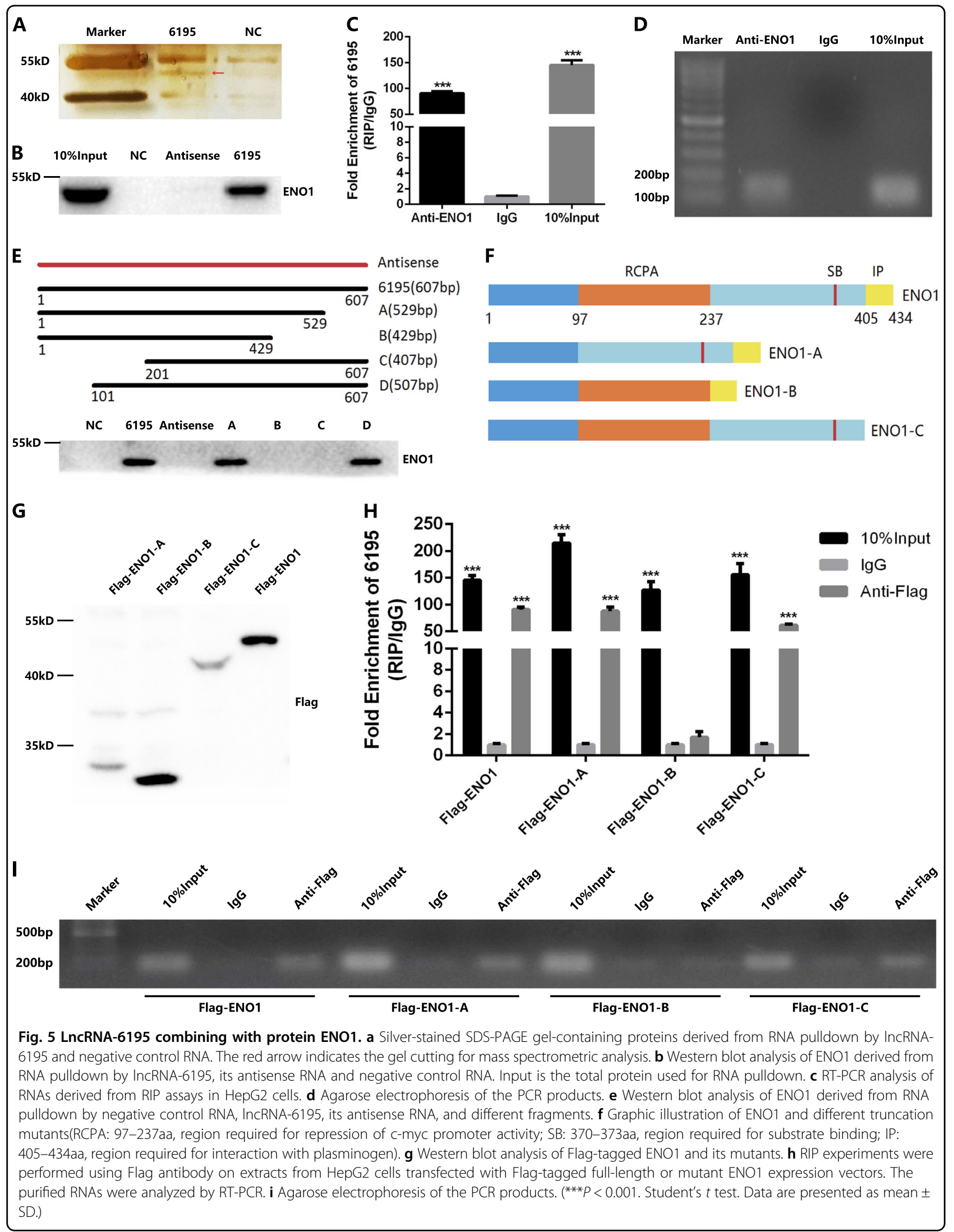


Table 2 Mass spectrometry analysis of the proteins pulled down by IncRNA-6195

\begin{tabular}{lccc}
\hline Protein hits & Mass & Score & emPAI \\
\hline a-Enolase & 47,481 & 182 & 0.76 \\
Elongation factor 1-a1 & 504,51 & 43 & 0.07 \\
Keratin, type II cytoskeletal 5 & 62,568 & 47 & 0.06 \\
Transketolase & 68,519 & 31 & 0.04 \\
Heat-shock cognate 71 kDa protein & 71,082 & 32 & 0.04 \\
Peroxisome biogenesis factor 1 & 143,804 & 37 & 0.02 \\
\hline
\end{tabular}

emPAl exponentially modified protein abundance index

binding region. The deletion mutants included the following fragments of lncRNA-6195: A (nucleotides 1-529), B (nucleotides 1-429), C (nucleotides 201-607), and D (nucleotides 101-607). The results indicated that ENO1 interacted with the region between nucleotides 101 and 529 of lncRNA-6195 (Fig. 5e).

We also constructed a series of FLAG-tagged deletion mutants of ENO1 and performed a RIP assay to map the lncRNA-6195-binding domain. The deletion mutants included ENO1-A, in which the 97-237-amino acid (aa) region, required for the repression of the c-myc promoter activity, was deleted; ENO1-B, in which the 237-405-aa region between the cleavage sites of ENO1-A and ENO1$\mathrm{C}$, containing the region (370-373 aa) required for substrate binding, was deleted; and ENO1-C, in which the 405-434-aa region, required for the interaction with plasminogen, was deleted (Fig. 5f, g). The assay data demonstrated the binding of lncRNA-6195 to the 237-405-aa region of ENO1 (Fig. 5h, i), which suggested that lncRNA-6195 might function by regulating the enzyme activity of ENO1.

\section{LncRNA-6195 can inhibit the enzymatic activity of ENO1}

Recent studies have demonstrated that IncRNA can interact with proteins through several modes of action, such as the modulation of protein function, regulation of protein-protein interactions, and direct localization within cellular compartments ${ }^{26}$. ENO1 is a multifunctional protein; it plays a role as a key glycolytic enzyme in the cytoplasm while serving as a plasminogen receptor on the surface of cells ${ }^{14}$. To investigate the molecular function of the IncRNA-6195-ENO1 ribonucleoprotein (RNP), the protein expression levels of ENO1 were measured by western blotting in different cell lines. The results showed no significant changes in the expression levels of ENO1 when IncRNA-6195 was upregulated in HepG2 and L02 cells or downregulated in HepG2/6195 cells, compared with those in their corresponding control groups (Fig. 6a). Subsequently, the distribution of ENO1 and lncRNA-6195 was studied in cells.
Both ENO1 and lncRNA-6195 were mainly located in the cytoplasm, with little detected in the nucleus in HepG2 and L02 cells (Fig. 6b, c), and almost no ENO1 was detected in the cell membrane in HepG2 cells. Additionally, the localization and expression levels did not significantly change in lncRNA-6195-upregulated cells (Fig. 6d). Lastly, the enzyme activity of ENO1, as well as glucose consumption and lactate production were determined. The data showed that the enzyme activity of ENO1 was reduced in lncRNA-6195-overexpressing L02 and HepG2 cells (Fig. 6e) and increased in lncRNA-6195downregulated HepG2/6195 cells (Fig. 6h). Consistently, the glucose consumption and lactate production were both reduced in IncRNA-6195-upregulated L02 and HepG2 cells (Fig. 6f, g) and increased in lncRNA-6195downregulated HepG2/6195 cells (Fig. 6i, j).

To further confirm that the RNA-protein interaction between lncRNA-6195 and ENO1 is the key antitumor function of lncRNA-6195, its deletion mutant B (Fig. 5e) was overexpressed in HepG2 cells transfected with the pcB plasmid (Supplementary Fig. S3C, D). The results showed that the pc-B transfection had no significant effect on the proliferation of HepG2 cells, as assessed by the CCK-8 assay (Fig. 6k), and there were no significant changes in the glucose consumption and lactate production in lncRNA-6195 deletion mutant B-overexpressing HepG2 cells (Fig. 6l, m).

Based on the above results, we suggest that lncRNA6195 can repress the HCC progression by combining with ENO1 and inhibiting its enzymatic activity.

\section{Discussion}

HCC is a worldwide disease with a very low 5-year survival rate ${ }^{1}$. There are numerous alterations in DNA, RNA, and proteins that enable cancer cells to immortally replicate, resist cell death, invade, and metastasize $e^{27}$. Recently, emerging evidence has greatly advanced our understanding of essential roles of lncRNAs in the pathogenesis and progression of $\mathrm{HCC}^{28-30}$. Researchers have found that $\mathrm{HBx}$ can alter the expression of lncRNAs, many of which have been suggested to be closely related to $\mathrm{HCC}^{11-13}$. Although hundreds of IncRNAs have been functionally characterized, the vast majority still remain to be studied. Our previous study has found that IncRNAs were aberrantly expressed in $\mathrm{HBx}$-overexpressing L02 cells. Expression of lncRNA-6195 was one of the most significantly altered in $\mathrm{LO} 2 / \mathrm{HBx}$ cells. In this study, we demonstrated that IncRNA-6195, whose function had never been investigated before, could inhibit the HCC growth in vitro and in vivo.

Recent studies have shown that lncRNAs can regulate the HCC growth in a variety of ways. In particular, lncRNAs induce many important cancer phenotypes through their interactions with other cellular 


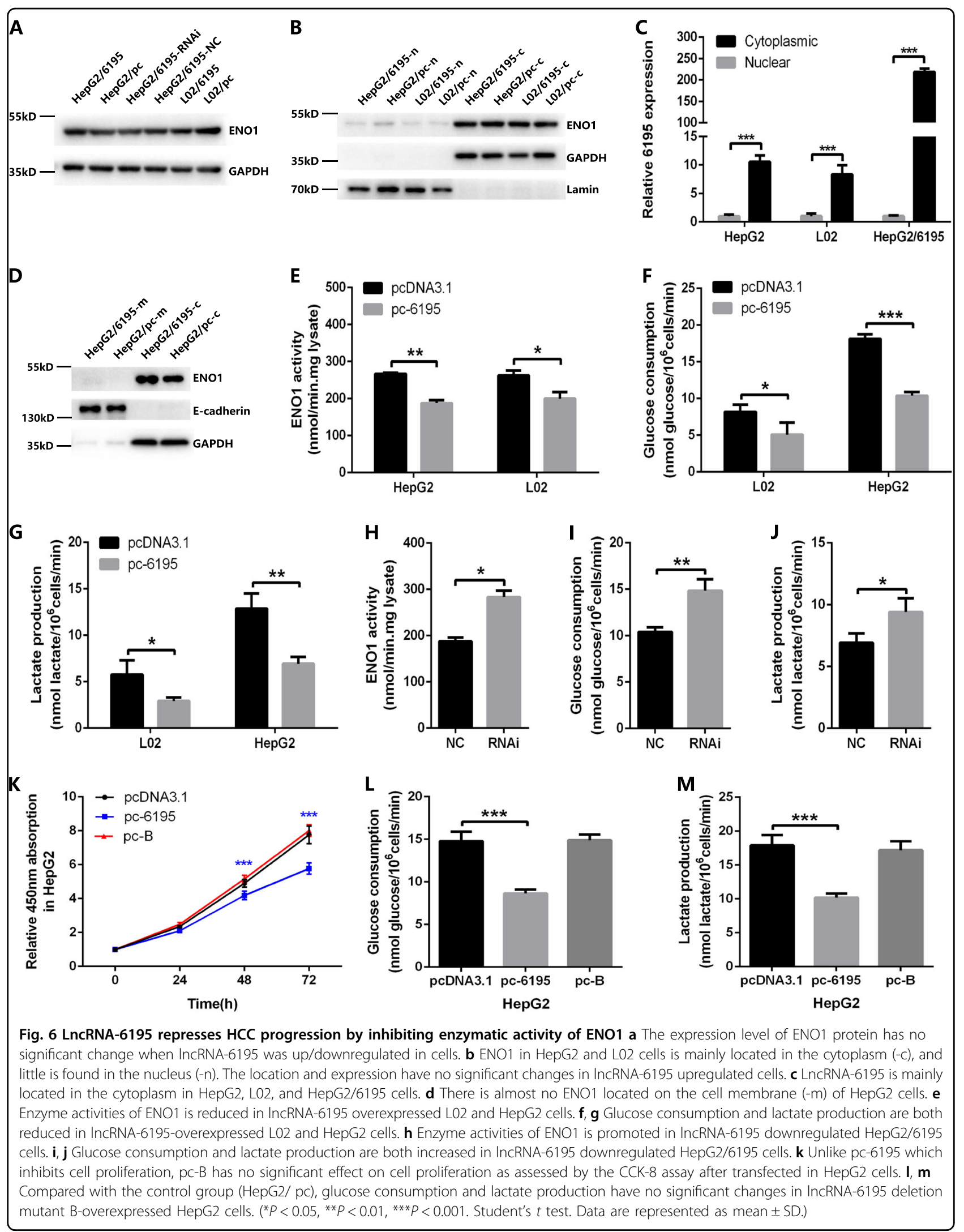




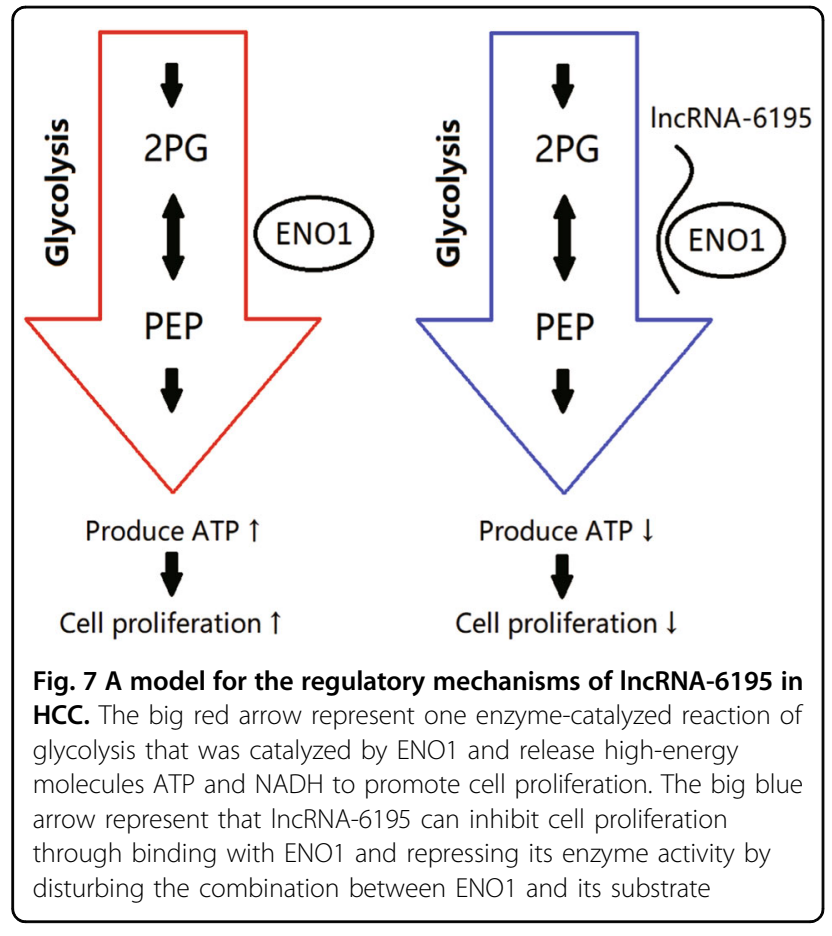

macromolecules, including DNA, proteins, and $\mathrm{RNA}^{26}$. After an unsuccessful search of all commonly used databases in an attempt to predict potential nucleotide sequences that could combine with lncRNA-6195, we speculated that IncRNA-6195 might repress the HCC growth by interacting with proteins. The RNA pull-down assay and mass spectrometry revealed that lncRNA-6195 could combine with the ENO1 protein. ENO1 is a multifunctional protein that has been implicated in several critical biological progresses in cancer, including the proliferation, migration, and invasion. In the cytoplasm, ENO1 catalyzes not only the transformation of 2PG to PEP during glycolysis but also the reverse conversion of PEP to 2PG during glycogen synthesis. By participating in anaerobic glycolysis (Warburg effect) and providing ATP, ENO1 is thought to promote cancer development and progression $^{31}$. On the cell surface, ENO1 binding to plasminogen results in enhanced plasminogen activation, localization of the plasmin proteolytic activity on the cell surface, and protection of plasmin from $\alpha-2$ antiplasmin $^{32,33}$. The plasminogen activation system is involved in cancer cell invasion and metastasis ${ }^{34-36}$. Recent studies have demonstrated that ENO1 is overexpressed in HCC tissue, which is correlated with the degree of tumor differentiation and progression ${ }^{18,37}$. The knockdown of ENO1 expression with a small interfering RNA significantly inhibited the proliferation of an HCC cell line ${ }^{17}$, whereas upregulation of the activity of the glycolytic enzyme ENO1 could promote the occurrence and development of cancer ${ }^{38}$.

As we found in this study, lncRNA-6195 did not affect the expression level of ENO1, and both lncRNA-6195 and ENO1 were mainly located in the cytoplasm. Their intracellular distribution suggested that the mechanism of action of the lncRNA-6195-ENO1 RNP might be associated with the enzyme activity. The data demonstrated that lncRNA-6195 could reduce the enzyme activity of ENO1. Further research led to the identification of the binding domain of ENO1 as the 237-405-aa region, which contains the substrate-binding site. This finding implies that IncRNA-6195 may repress the enzyme activity of ENO1 through the disruption of ENO1-substrate interaction.

Collectively, our results reveal that enhanced expression of lncRNA-6195 can reverse the progression of HCC via lncRNA-6195 combining with ENO1 and inhibiting its enzymatic activity (Fig. 7). These findings suggest new therapeutic strategies for the prevention and treatment of HCC.

\section{Materials and methods \\ Patient samples}

In this study, HBV-related HCC specimens and adjacent non-tumorous liver tissues were collected from 47 patients who were pathologically diagnosed with HBVrelated HCC between July 2014 and April 2015 at the XiangYa Hospital, Central South University (Changsha, China). Patients who received radiotherapy, embolotherapy, or chemotherapy were excluded. All patients were followed up for 24 months post surgery. OS was defined as the interval between tumor resection and death or the last follow-up examination. The patients' clinicopathological characteristics are summarized in Supplementary Table S1. The study was approved by the Ethics Committee of the XiangYa Hospital, and written informed consent was obtained from all patients.

\section{RNA isolation andRT-PCR}

Total RNA was extracted from HCC and adjacent liver tissues or cultured cells using the EZNA total RNA kit I (Omega, USA), while nuclear and cytoplasmic RNA was extracted from cultured cells using the PARIS kit (Life Technologies, USA) according to the manufacturer's instructions. Subsequently, RNA was reverse-transcribed into cDNA using the PrimeScript RT reagent kit with gDNA Eraser (Takara, Japan). RNA expression levels were measured by qRT-PCR on the ABI 7500 Fast real-time PCR platform using SYBR ${ }^{\circledast}$ Premix Ex Taq II (Takara, 
Japan). Relative quantification was performed using the $2^{-\Delta \Delta \mathrm{Ct}}$ method. The primers used are listed in Supplementary Table $\mathrm{S} 2$.

\section{Cell lines}

The Huh7, HepG2, 293T, and L02 cell lines were purchased from the Cell Bank of the Chinese Academy of Sciences (Shanghai, China). Huh7, HepG2, and 293T cells were maintained in Dulbecco's modified Eagle's medium (Gibco, USA) supplemented with 10\% fetal bovine serum (FBS; Gibco, USA). L02 cells were maintained in RPMI 1640 medium (Gibco, USA) supplemented with $10 \%$ FBS. All cells were incubated at $37^{\circ} \mathrm{C}$ in an incubator with $5 \%$ $\mathrm{CO}_{2}$.

\section{Plasmid and lentiviral constructions}

The lncRNA-6195 expression plasmid vector (pc-6195) and the lncRNA-6195 part B expression plasmid vector (pc-B) were constructed by inserting a full-length lncRNA-6195 or a part B lncRNA-6195 fragment (Supplementary Fig. S3B) into the BamHI/EcoRI site of pcDNA3.1. The FLAG-tagged full-length ENO1 protein and ENO1 deletion mutants were constructed by inserting a full-length $E N O 1$ or fragments of $E N O 1-\mathrm{A} / \mathrm{B} / \mathrm{C}$ mutants (Fig. 5f) into the BamHI/EcoRI site of pcDNA3.1. Short hairpin RNAs (shRNAs) against lncRNA-6195 were designed (Gene Pharma, China) and cloned into the BamHI/EcoRI sites of Lenti-X expression vectors (Clontech, USA). Recombinant pLVX-shRNA vectors and the Lenti-X HTX packaging system (Clontech, USA) were used to produce high-titer lentivirus in 293T packaging cells. The sequences are described in Supplementary Table S2.

\section{Cell transfection and RNA interference}

The lncRNA-6195 expression vector, control plasmid vector, and FLAG-tagged full-length or mutant ENO1 expression vectors were transfected into cells using the Fugene 6 reagent (Promega, USA) according to the manufacturer's instructions. The expressing cells were established using G418. The IncRNA-6195 shRNA or control shRNA was transfected into cells using polybrene (Genomeditech, China). Stably transfected cell lines were selected using puromycin, and the expression level of lncRNA-6195 was confirmed by qRT-PCR.

\section{Cell proliferation assay}

Cells were seeded in 96-well plates at a density of 5000 cells per well in $100 \mu \mathrm{L}$ of complete medium. At the indicated time points, $10 \mu \mathrm{L}$ of the Cell Counting Kit-8 (CCK-8) reagent (Beyotime, China) was added to each well, and the plate was incubated at $37^{\circ} \mathrm{C}$ for an additional $1 \mathrm{~h}$. The absorbance in each well was measured at $450 \mathrm{~nm}$ using a microplate reader.

\section{Colony formation assay}

Cells were seeded in 6-well plates at a density of 500 cells per well. After approximately 2 weeks, the culture medium was removed, cells were stained with crystal violet, and colonies that had more than 50 cells were counted.

\section{Cell cycle and cell apoptosis assay}

The cell cycle and the cell apoptosis were measured on flow cytometer (FACSCalibur, BD Biosciences, San Jose, CA) platform using Cell Cycle Analysis kit (Beyotime, China) or Annexin V-FITC Apoptosis Detection kit (KeyGen Biotech, China). All data were analyzed by the FlowJo X software.

\section{Proteins isolation and western blot analysis}

Total proteins were extracted from cultured cells using cell lysis buffer for western and IP (Beyotime). Membrane proteins and nuclear and cytoplasmic proteins were extracted from cultured cells using a Mem-PER Plus membrane protein extraction kit (Thermo Scientific, USA) and NE-PER nuclear and cytoplasmic extraction reagents (Thermo Scientific), respectively, according to the manufacturer's instructions. Proteins were separated by $8-12 \%$ SDS-PAGE and transferred to polyvinylidene difluoride membranes $(0.25 \mu \mathrm{m}$; Millipore). The membranes were blocked with $5 \%$ bovine serum albumin in Tris-buffered saline with Tween-20 (TBST) for $1 \mathrm{~h}$, then washed with TBST, and incubated with primary antibodies overnight at $4{ }^{\circ} \mathrm{C}$. After subsequent incubation with horseradish peroxidase-conjugated secondary antibodies for $1 \mathrm{~h}$ at $25^{\circ} \mathrm{C}$, signals were visualized using an enhanced chemiluminescence method (Bio-Rad). The relative band intensity was measured using the Image Lab software. Anti-tubulin and glyceraldehyde 3-phosphate dehydrogenase antibodies were obtained from Auragene Bioscience (Changsha, China). The FLAG antibody was obtained from Sigma-Aldrich (St. Louis, MO, USA). Other antibodies used were obtained from Abcam (Cambridge, MA, USA).

\section{Animal experiments}

$\mathrm{BALB} / \mathrm{C}$ nude mice (male, 4-week-old) were purchased from the Hunan Technology Transfer Center Experimental Animal Division of the Chinese Academy of Sciences (Hunan, China). All animal experiments were conducted in compliance with the regulations of the Institutional Animal Care and Use Committee of the Department of Laboratory Animals of CSU in China. To investigate the effects of IncRNA-6195 on tumorigenesis in vivo, we subcutaneously injected HepG2/6195 or HepG2/pc cells (stably transfected with pc-6195 or pcDNA3.1, respectively) into BALB/C nude mice $(n=6)$. The volumes of subcutaneous tumors were measured 
every 6 days after implantation. After 24 days, the mice were sacrificed to harvest tumor tissue for analysis. Tissue sections were stained with HE or subjected to Ki-67 immunohistochemical staining.

\section{Immunohistochemistry}

IHC for Ki-67 was performed on paraffin-embedded tumor sections using a primary antibody against Ki-67 (Abcam, USA) and a goat anti-rabbit IgG horseradish peroxidase-conjugated secondary antibody (Abcam, USA). Proteins were visualized in situ with 3,3'-diaminobenzidine, and photographs were analyzed using the Image-Pro Plus 6.0 software.

\section{RNA pull-down assay}

LncRNA-6195, its A-D fragments, and antisense RNA were transcribed from the pcDNA3.1-6195 vector using the TranscriptAid T7 high-yield transcription kit. RNA-protein pull-down experiments were performed using the Pierce ${ }^{\mathrm{Ta}}$ magnetic RNA-protein pull-down kit according to the manufacturer's instructions. Retrieved proteins were separated by $12 \%$ SDS-PAGE, then silverstained using the Pierce silver stain kit, and specific bands were cut out and analyzed by mass spectrometry using the Mascot software. All the kits used in this assay were obtained from Thermo Fisher Scientific (Waltham, MA, USA).

\section{RIP assay}

RIP experiments were performed using the Magna RIP ${ }^{\mathrm{mm}}$ RNA-binding protein immunoprecipitation kit (Millipore, Danvers, MA, USA) according to the manufacturer's instructions. The anti-ENO1 antibody for the RIP assay was obtained from Abcam. RT-PCR was used to examine whether co-precipitated RNA contained lncRNA-6195. The primers used are presented in Supplementary Table S2.

\section{Glucose consumption and lactate production}

Cells were seeded in 6-well plates at a density of $5 \times 10^{5}$ cells per well. After $24 \mathrm{~h}$ of incubation, the medium was collected and centrifuged at $2000 \mathrm{rpm}$ for $5 \mathrm{~min}$ to remove cell debris. Glucose and lactate levels were determined in the culture medium using an automatic biochemical analyzer (Beckman, Germany). Cell-free medium was used as the blank control.

\section{Enzyme activity assay}

The enzymatic activity of ENO1 was measured in different cell lines using an enolase activity assay kit (SigmaAldrich, St Louis, MO, USA) according to the manufacturer's instructions.

\section{Statistical analysis}

Statistical analysis was performed using the SPSS 24.0 and GraphPad Prism 6 software. Each experiment was performed at least three times. The expression of lncRNA-6195 and HBx in patient HCC tissue and tumoradjacent tissue was compared by a paired sample $t$ test. Survival curves were calculated using the Kaplan-Meier method and a log-rank test. The $\chi^{2}$ test, Fisher's exact test, and Student's $t$ test were used for comparison between groups. Normally distributed data are expressed as the mean \pm standard deviation (SD). All $P$-values were twosided, and $P<0.05$ was accepted as statistically significant.

\section{Acknowledgements}

This work was supported by grant from International Scientific and Technology Cooperation Program of China (No. 2015DFA31490), National Natural Sciences Foundation of China (Nos. 81272253, 81700561, and 81402623), and Natural Sciences Foundation of Hunan province (No. 2017JJ3496)

\section{Author details}

${ }^{1}$ Department of Infectious Diseases, Hunan Key Laboratory of Viral Hepatitis, Xiangya Hospital, Central South University, Changsha, China. ${ }^{2}$ Department of Blood Transfusion, Xiangya Hospital, Central South University, Changsha, China. ${ }^{3}$ Department of Surgery, University of Pittsburgh, Pittsburgh, PA, USA

\section{Conflict of interest}

The authors declare that they have no conflict of interest.

\section{Publisher's note}

Springer Nature remains neutral with regard to jurisdictional claims in published maps and institutional affiliations.

Supplementary Information accompanies this paper at (https://doi.org/ 10.1038/s41419-018-1231-4).

Received: 27 June 2018 Revised: 20 November 2018 Accepted: 21 November 2018

Published online: 05 December 2018

\footnotetext{
References

1. Bray, F. et al. Global cancer statistics 2018: GLOBOCAN estimates of incidence and mortality worldwide for 36 cancers in 185 countries. CA Cancer J. Clin. 0, 1-31 (2018).

2. Levrero, M. \& Zucman-Rossi, J. Mechanisms of HBV-induced hepatocellular carcinoma. J. Hepatol. 64, S84-S101 (2016).

3. Zhou, H. Y., Luo, Y., Chen, W. D. \& Gong, G. Z. Hepatitis B virus mutation may play a role in hepatocellular carcinoma recurrence: a systematic review and meta-regression analysis. J. Gastroenterol. Hepatol. 30, 977-983 (2015).

4. Ponting, C. P., Oliver, P. L. \& Reik, W. Evolution and functions of long noncoding RNAs. Cell 136, 629-641 (2009).

5. Yuan, J. H. et al. A long noncoding RNA activated by TGF-beta promotes the invasion-metastasis cascade in hepatocellular carcinoma. Cancer Cell 25, 666-681 (2014).

6. Yuan, S. X. et al. Long noncoding RNA DANCR increases stemness features of hepatocellular carcinoma by derepression of CTNNB1. Hepatology 63, 499-511 (2016)

7. Yang, F. et al. Long noncoding RNA high expression in hepatocellular carcinoma facilitates tumor growth through enhancer of zeste homolog 2 in humans. Hepatology 54, 1679-1689 (2011).

8. Yuan, S. X. et al. Long noncoding RNA associated with microvascular invasion in hepatocellular carcinoma promotes angiogenesis and serves as a predictor
} 
for hepatocellular carcinoma patients' poor recurrence-free survival after hepatectomy. Hepatology 56, 2231-2241 (2012).

9. Li, S. et al. The long non-coding RNA TP73-AS1 modulates HCC cell proliferation through miR-200a-dependent HMGB1/RAGE regulation. J. Exp. Clin. Cancer Res. 36, 51 (2017).

10. Moyo, B., Nicholson, S. A. \& Arbuthnot, P. B. The role of long non-coding RNAs in hepatitis B virus-related hepatocellular carcinoma. Virus Res. 212, 103-113 (2016).

11. Huang, J. F. et al. Hepatitis B virus X protein ( $\mathrm{HBX}$ )-related long noncoding RNA (IncRNA) down-regulated expression by HBx (Dreh) inhibits hepatocellular carcinoma metastasis by targeting the intermediate filament protein vimentin. Hepatology 57, 1882-1892 (2013).

12. Hu, J. J. et al. HBX-upregulated IncRNA UCA1 promotes cell growth and tumorigenesis by recruiting EZH2 and repressing p27Kip1/CDK2 signaling. Sci. Rep. 6, 23521 (2016).

13. LV, J. et al. Long non-coding RNA Unigene56159 promotes epithelial-mesenchymal transition by acting as a ceRNA of miR-140-5p in hepatocellular carcinoma cells. Cancer Lett. 382, 166-175 (2016).

14. Pancholi, V. Multifunctional alpha-enolase: its role in diseases. Cell. Mol. Life Sci. 58, 902-920 (2001).

15. Song, $Y$. et al. Alpha-enolase as a potential cancer prognostic marker promotes cell growth, migration, and invasion in glioma. Mol. Cancer 13, 65 (2014).

16. Zhan, P. et al. a-enolase promotes tumorigenesis and metastasis via regulating AMPK mTOR pathway in colorectal cancer. Mol. Carcinog. 56, 1427-1437 (2017).

17. Hamaguchi, T. et al. Glycolysis module activated by hypoxia-inducible factor lalpha is related to the aggressive phenotype of hepatocellular carcinoma. Int. J. Oncol. 33, 725-731 (2008).

18. Takashima, M. et al. Overexpression of alpha enolase in hepatitis C virusrelated hepatocellular carcinoma: association with tumor progression as determined by proteomic analysis. Proteomics 5, 1686-1692 (2005).

19. Kuang, X. Y., Li, N., Fu, Y. M., Li, J. \& Fan, X. G. Expression profiles of long noncoding RNAs in human liver cell line $\mathrm{LO} 2$ with stable expression of hepatitis $B$ x gene. Zhonghua Gan Zang Bing. Za Zhi 24, 417-421 (2016).

20. Chen, R. C. et al. Integrated analysis of microRNA and mRNA expression profiles in HBx-expressing hepatic cells. World J. Gastroenterol. 23, 1787-1795 (2017).

21. Lin, M. F., Jungreis, I. \& Kellis, M. PhyloCSF: a comparative genomics method to distinguish protein coding and non-coding regions. Bioinformatics 27, i275-i282 (2011).

22. Wang, L. et al. CPAT: Coding-Potential Assessment Tool using an alignmentfree logistic regression model. Nucleic Acids Res. 41, e74 (2013).
23. Vizcaíno, J. A. et al. 2016 update of the PRIDE database and its related tools Nucleic Acids Res. 44, D447-D456 (2016).

24. Bazzini, A. A. et al. Identification of small ORFs in vertebrates using ribosome footprinting and evolutionary conservation. EMBO J. 33, 981-993 (2014).

25. Lee, S. et al. Global mapping of translation initiation sites in mammalian cells at single-nucleotide resolution. Proc. Natl. Acad. Sci. USA 109, E2424-E2432 (2012).

26. Schmitt, A. M. \& Chang, H. Y. Long noncoding RNAs in cancer pathways. Cancer Cell 29, 452-463 (2016).

27. Hanahan, D. \& Weinberg, R. A. Hallmarks of cancer: the next generation. Cell 144, 646-674 (2011).

28. Guttman, M. \& Rinn, J. L. Modular regulatory principles of large non-coding RNAs. Nature 482, 339-346 (2012).

29. Shi, L., Peng, F., Tao, Y., Fan, X. \& Li, N. Roles of long noncoding RNAs in hepatocellular carcinoma. Virus Res. 223, 131-139 (2016).

30. Peng, L. et al. The emergence of long non-coding RNAs in hepatocellular carcinoma: an update. J. Cancer 9, 2549-2558 (2018).

31. Vander Heiden, M. G., Cantley, L. C. \& Thompson, C. B. Understanding the Warburg effect: the metabolic requirements of cell proliferation. Science 324, 1029-1033 (2009).

32. Miles, L. A. et al. Role of cell-surface lysines in plasminogen binding to cells: identification of a-enolase as a candidate plasminogen receptor. Biochemistry 30, 1682-1691 (1991).

33. Redlitz, A., Fowler, B. J., Plow, E. F. \& Miles, L. A. The role of an enolase-related molecule in plasminogen binding to cells. Eur. J. Biochem. 227, 407-415 (1995).

34. Andreasen, P. A., Egelund, R. \& Petersen, H. H. The plasminogen activation system in tumor growth, invasion, and metastasis. Cell. Mol. Life Sci. 57, 25-40 (2000).

35. Choong, P. F. \& Nadesapillai, A. P. Urokinase plasminogen activator system: a multifunctional role in tumor progression and metastasis. Clin. Orthop. Relat. Res. 415, S46-S58 (2003).

36. Reuning, $U$. et al. Multifunctional potential of the plasminogen activation system in tumor invasion and metastasis (review). Int. J. Oncol. 13, 893-906 (1998).

37. Jiang, B. H., Agani, F., Passaniti, A. \& Semenza, G. L. V-SRC induces expression of hypoxia-inducible factor 1 (HIF-1) and transcription of genes encoding vascular endothelial growth factor and enolase 1: involvement of HIF-1 in tumor progression. Cancer Res. 57, 5328-5335 (1997).

38. Chen, S. et al. WW domain-binding protein 2 acts as an oncogene by modulating the activity of the glycolytic enzyme ENO1 in glioma. Cell Death Dis. 9, 347 (2018). 